\title{
A Versatile Personal Assistant Dedicated to B/VIP
}

\author{
Paul Costache, Simona Riurean ${ }^{*}$ and Sebastian Rosca
}

\author{
University of Petrosani, Petrosani 332006, Romania
}

\begin{abstract}
Today, there are different systems on the market that aim to help Blind or Visually Impaired People (B/VIP) to gain more independency and self-trust. From the classic white cane to various intelligent devices to guide people with vision problems, most of the systems are expensive, difficult to be used or with low efficiency. The subject is a very important one because, according to the World Health Organization, there are approximately 2.2 billion people in the world that have a vision impairment or blindness. Thus, the purpose of this work is to make a short survey on solutions available on the market, in order to develop a low-cost, efficient and easy-to-use system for both indoor and outdoor use. The versatile personal assistant dedicated to B/VIP aims to enhance their independence and also improved outdoor navigation. The smart warning system addressed here is an innovative device aiming to detect obstacles with the help of ultrasonic sensors and warning of their presence by means of a buzzer or vibration. The system, when used outdoor, also gives the possibility to send short message to the B/VIP's own assistant, in the form of an SMS with the exact location of the B/VIP. Moreover, the SMS' content can easily create the optimal route to the B/VIP's location with the Google Maps support for a fast-time assistance.
\end{abstract}

Keywords: Blind and Visually Impaired People, ultrasonic reflexive sensor, SMS warning.

\section{INTRODUCTION}

Surrounding environmental signals are very important for both Blind and Visually Impaired People (B/VIP), therefore acoustic feedback must be carefully used since is appropriate in all cases, especially when the acoustic feedback is continuous. Although in the well-known macro-navigation scenarios, the speech output (like the GPS system provided by Google maps or installed into the cars navigation systems used by drivers) proved to be the most agreed, reasonable choice, the B/VIP do not feel comfortable to use [1]. On the other hand, different B/VIP interpret audio messages differently or routes may differ if navigation instructions are given by sighted persons instead of B/VIP [2]. An alternative is the message received by B/VIP from a vibrational feedback systems used for navigation purposes in different ways, going from vibrating headbands [3] to systems tapped on shoulders [4]. The main subject of this paper refers to the need of improved mobility for blind people or those with visual impairments. According to the World Health Organization, there are approximately 2.2 billion B/VIP in the world [5], this subject thus being an important one with many technological implementations and challenges.

According to many research projects conducted on different groups of people with major impairments of vision or even completely blind, the most difficult problem they encountered is finding the best way when they move in an unknown environment (or even in a known one) inside or outside of a building $[6,7,8]$.

Address correspondence to this article at the University of Petrosani, Petrosani 332006, Romania; Tel: 0000-0002-5283-6374; E-mail: sriurean@yahoo.com
The white cane dedicated to B/VIP is a white aluminum stick with a handle at one end and a silencer on the other end. Although used since 1920s, the white cane has been improved by George A. Bonham in 1930 and became internationally used after the second world war [9]. Many smart canes have been developed worldwide since then with different new technologies embedded, as for example a smart walking stick with ultrasonic sensor used to detect the obstacle with the help of a camera [10].

The guide dogs are assistance dogs trained to lead B/VIP around obstacles in order to move safely and efficiently from one place to another. The first dogs training schools have been settled in Germany during the World War I, aiming to enhance the mobility of returning veterans who were B/VIP in combat. The $\mathrm{B} / \mathrm{VIP}$ are no longer forced to walk in small steps, hitting each potential obstacle with the white can. The dog can quickly calculate and follow a route that does not seem to have obstacles (from one intersection to another it can see if the route is free and maintains an alert and uninterrupted pace of walking) [11].

The Smart glasses for the blind are used to improve the mobility of people who have severe vision problems, therefore not totally blind. With these glasses, the blind can move safely both inside and outside. The glasses consist of a helmet and a transparent screen with two small video cameras, a GPS module, a gyroscope and a compass. The device has a video camera mounted on the frame and, with the support of a software, the images are projected on the screen. The glasses combine the information transmitted through an infrared beam and a normal 
video camera. A computer then works on processing data and displaying them in much brighter contours [12].

The Tandem Access app helps visually impaired people to travel by subway without the help of an attendant and without being dependent by others. This application is based on Bluetooth wireless communication technology, giving access to the guidance system even if the phone does not have a signal or a GPS location. The guidance devices emit an audible signal and communicate with the smartphone through the application that sends to the blind messages (read by voice-over) with guidance on the key points of the subway stations (intersections, stairs, etc.) [13].

The Cydalion App is a new Augmented Reality (AR) smartphone app that makes navigating in an unknown environment much easier for the visually impaired. The application uses sound and vibration to enhance the user's understanding of the environment and inform him about the obstacles around him. The device is not based on GPS signal or any other external signal so it does not depend on other external factors for optimal operation (e.g. GPS signal that is not available indoors or in the underground environment) [14].

Many indoor positioning and guiding systems based on Visible Light Communication (VLC) for Visually Impaired People have also been developed. One of them, a dedicated indoor positioning and guiding system (IPGS) is with an optical transmitter with LED embedded into the illumination fixture and a low cost, handy, wearable optical receiver with solar panel intended to be used by VIP when accessing public institutions as schools, museums, indoor stations as underground metro or airports with a dedicated infrastructure to support IPGS [15].

In the paper "Fundamentals of Indoor VLP: Providing Autonomous Mobility for Visually Impaired People" the authors show how VLC or Optical Camera Communications (OCC) are used as guidance technologies for VIP on indoor scenarios, using universally available smartphone cameras as receiver [16].

A system based on Android technology dedicated to VIP with a dedicated application that helps the user to open any app and call a contact through voice commands. VIP can send speech messages to a mobile device that are immediately interpreted by the
Speech Recognition Engine (SRE) that converts speech into text for direct actions or make a voice call to a known person. The system is also equipped with a "Selendroid" app interface which enables the VIP to fetch the latest information from different web servers. The newest data retrieved by the "Selendroid" architecture includes weather report, information related to transport and news update [17].

Following extended research, we discovered that, most of the existing systems on the market, dedicated to the B/VIP, are inefficient, obsolete or too expensive being therefore unaffordable for most of the B/VIP. There are, however, a number reliable solutions already successfully used by B/VIP, that offer fast and safe orientation in macro or micro navigation scenarios. Based on the current advanced technology, tremendous possibilities of new ideas implementation are possible.

Analyzing the various problems encountered by B/VIP when move outdoor or even indoor, three major requirements for reliable guiding system developed by us are identified: management of distance measured by sensors, accuracy of the distance measured between B/VIP and the surrounding objects/obstacles and the third one, precise localization of the B/VIP in real time in order to assist them in due time.

A general representation of the guiding system developed by us for a high accuracy position and guiding indoor and outdoor with the specific modules, is presented in Figure 1.

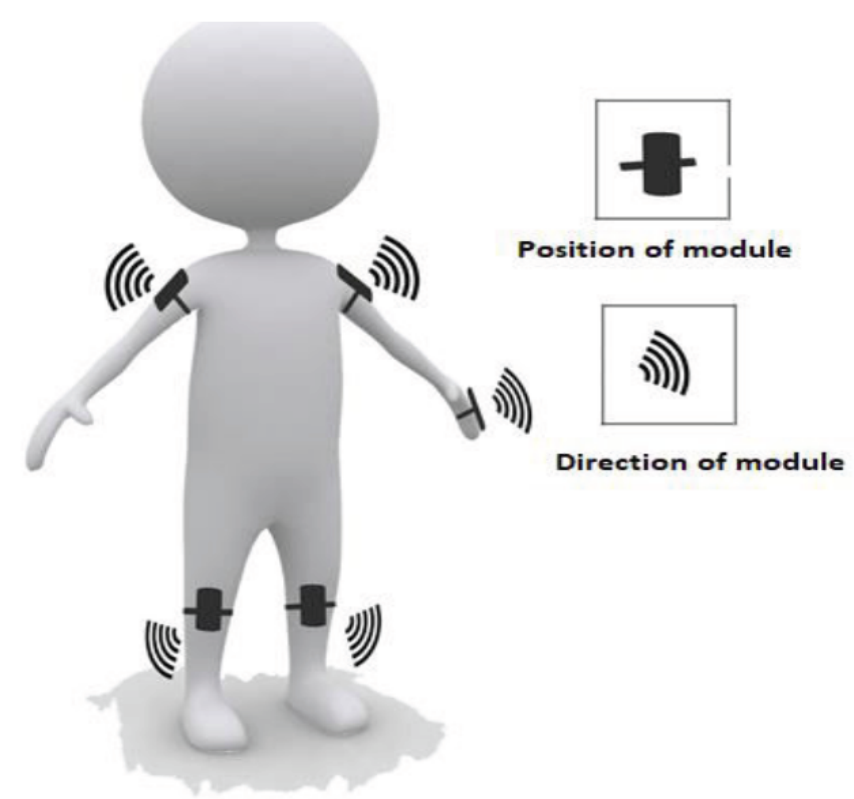

Figure 1: Position and location of modules on B/VIP. 
The system consists of five independent modules. Each module has its own functionality embedded with orientation in different direction, as follows:

- A module that scans the environment in every direction, placed on a glove in the palm of the B/VIP.

- A module placed on the left arm facing the side required, to prevent reading the obstacles placed on the left.

- A module placed on the right arm facing the necessary side to prevent reading the obstacles placed on the right side.

- A module located under the left knee to notify B/VIP about any possible obstacles that may occur in proximity.

- A module placed under the right knee to notify B/VIP about any obstacles that may arise in front.

\section{DISTANCE MANAGEMENT USING SENSORS}

When developing this project, in order to follow all the requirements regarding handy and low-cost device, the Arduino environment has been used. Arduino, an open-source company, produces both microcontrollerbased development boards and the software part dedicated to their operation and programming. Arduino boards use a variety of microprocessors and controllers. The boards are equipped with several sets of digital and analog pins, both input and output pins allowing interaction with different circuits, sensors or shields. Microcontrollers are typically programmed using $\mathrm{C}^{++}$programming language. In addition to classic compilation tools, Arduino offers an Integrated Development Environment (IDE) [18].

Two possible choices of sensors to be used in our project are Infrared (IR) or ultrasonic sensors.

The sensor consists of two components, an LED that emits light in the IR spectrum and a photodiode that receives the reflected waves. The IR sensor uses the IR waves to measure distances. It is based on the light wave emitted that measures the time until the light wave reaches the translator due to an obstacle reflection. To avoid interference with other external light sources, the LED light signal is modulated.

Ultrasonic sensors (US) used to measure distance work, as well, on the principle of reflected waves, this time sound waves. One US can detect other sensors operating nearby. Sound waves are emitted by the US and they are reflected back if there is an object in front of it. The US detects these waves and measures the time it takes between transmitting and receiving those sound waves. Distance is then estimated by the time interval between sensor and object.

Although USs are, for the most part, completely insensitive to hindering factors like: light, dust, smoke, mist, vapor and so on, they are not as good as IR at defining edges of an area.

With the aim of measuring the distance between the B/VIP and surrounding objects as possible identified obstacles that may be located in the user's path, we consider appropriate to use the Arduino development environment. It offers several tools to develop a versatile personal assistant dedicated to mobility of blind or visually impaired people: ultrasonic distance measurement sensors and distance sensors based on the speed of light.

For example, RPS-401A, RPS-412A or RPS-426A, some of the most technological advanced (but more expensive) sensors can be used for this aim are advanced ultrasonic sensors (AUSs) that can precisely measure the distance to an obstacle. Among the advantages of the new, AUSs over traditional sensors is the ability to detect and measure moving objects. Colors, shapes or surface's texture of the object does not affect these sensors, being capable to detect small objects over long distances. AUSs are also able to measure an approaching or withdrawing object. By using "non-contact" AUSs, distances can be measured without damage to the object, being easy to use. In many cases, AUSs can replace other traditional sensors when the environmental conditions make traditional sensors unusable [19].

However, in order to keep the costs of the entire project as low as possible, and due to a large availability, we chose to use the HC-SR04 a long range, ultrasonic reflexive sensor used for distance measurement or object detection. The measuring range is between $2 \mathrm{~cm}$ and $400 \mathrm{~cm}$ with a precision of 2-3 $\mathrm{mm}$ and an optimum angle of measurement of 15 degrees.

The module has an ultrasonic transmitter, a receiver and a control circuit, and the working principle is similar to that of bats and dolphins (echolocation).

HC-SR04 sends a sonic pulse of $40 \mathrm{KHz}$ in 8 cycles, to the Trig pin, which moves with the speed of 
sound through the air and will be received to the Echo pin, after its reflection off an obstacle. The Echo pine will show the time, in microseconds, of the received sound waves.

The calculation of the distance using the HC-SRO4 ultrasonic sensor is based on the difference between the time at which the sonic pulse was transmitted and the moment at which it is detected back, taking into account the speed of sound and using the well-known physics formula for space SPACE $=S P E E D^{*} T I M E$.

We can also express the time in microseconds, necessary for the signal to travel one centimeter, which has the value of $29.41 \mu \mathrm{s} / \mathrm{cm}$, knowing that the speed of sound is $340 \mathrm{~m} / \mathrm{s}$, with the formula:

$\frac{1}{340 \mathrm{~m} / \mathrm{s}} * \frac{10000000 \mu \mathrm{s}}{1 \mathrm{~s}} * \frac{1 \mathrm{~m}}{100 \mathrm{~cm}}=29,41 \mu \mathrm{s} / \mathrm{cm}$

\section{FAST IDENTIFICATION OF B/VIP LOCATION}

In order to locate B/VIP in real time, the Sim 808 Development Board module (GSM + GPRS + GPS) is used. This module incorporates the functions of a GSM module as well as a GPS module. By using it, the B/VIP location can be fast identified. Moreover, the B/VIP can also send SMS or initiate calls.

It is a very easy module to use because it is controlled with the help of AT commands through serial communication. AT commands are instructions used to control a modem. AT stands for Attention. Each command starts with the prefix "AT" or "at" and for this they are called "AT commands". Many of these commands are also used to control GSM / GPRS devices or cell phones.

\section{TOOLS USED FOR SYSTEM'S DEVELOPMENT}

According to the project's requirements and methods presented above we choose to use the ultrasonic sensor together with an Arduino PRO MINI board to measure the distance from any obstacles. To notify in real time the B/VIP of an obstacle existence, we use a BUZZER or a vibration motor. The intensity of the sounds / vibrations increases when B/VIP approach an obstacle being, therefore able to choose the warning mode through a switch, that changes the warning mode between sounds and vibrations.

The resulting device being a modular and portable one, the B/VIP can choose how to use it or where to wear it, having the possibility to make his own set according to his own needs. We believe that the device can be worn on any part of the body, for example on the front of the first to help the B/VIP to move inside and outside, but it can also be mounted in his own house to warn B/VIP when approaching surrounding objects with the module mounted on.

Because in a set of several devices worn simultaneously by the B/VIP or in case of mounting it on different objects/obstacles indoor, it is not necessary for the GPS module to be present. Therefore, we designed a basic device without the GPS tracking module which can be added as and extending (see Figure 3). The GPS module, that becomes active by pressing a button, activates the GPS location and

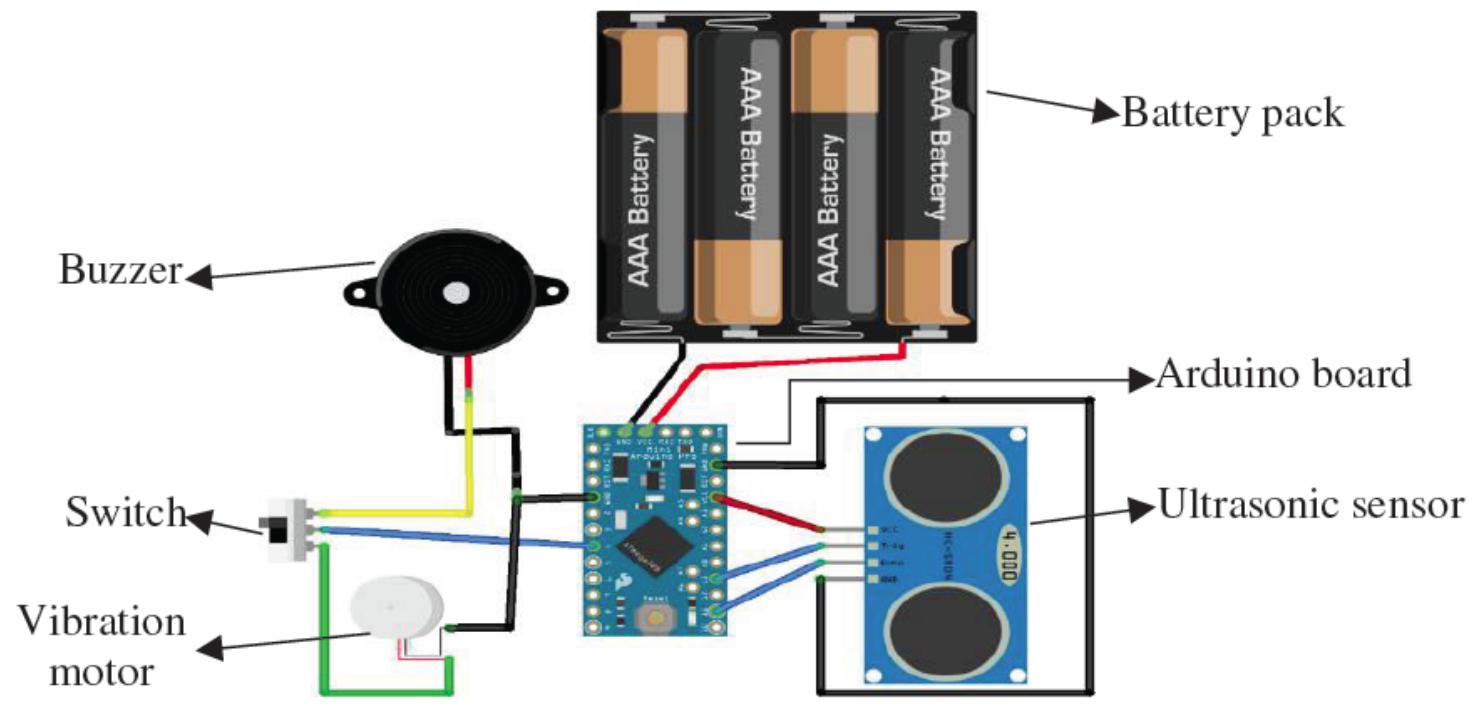

Figure 2: General diagram of the basic module placed onto a glove into B/VIP s palm. 
sends a SMS to a predetermined person (B/VIP assistant, for example) with his/her location to inform the assistant that the B/VIP needs help.

As the reference distance we chose the value of 500 millimeters. The device starts to warn the B/VIP when an object is less than 500 millimeters from it.

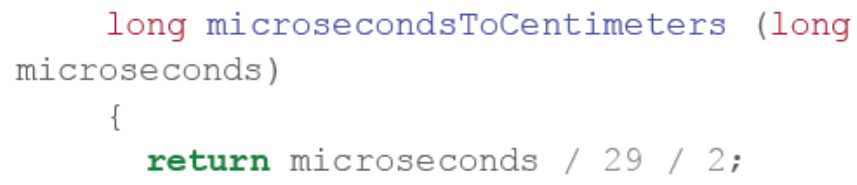

long microsecondsToCentimeters (long microseconds)

\{ return microseconds / 29 / 2;

In order for this entire system to properly work, we also need a suitable software. Using all the information presented above, the $\mathrm{C}^{++}$programming language has been used.

The distance calculation is done using the formulas presented in (2). In this case, the long microsecondToCentimeters function (long microseconds) is a long peak function while it can stay back or slow down and accept a long time parameter. The parameter is the amount of sonic pulse.

Distance formula used is:

distance $=\frac{\frac{\text { microseconds }}{S}}{2}$

where

$\mathrm{S}$ - is the speed of sound through the air (the value used is 29.41).
The mode of operation of this module is similar to that of the modules presented previously with the difference that when the B/VIP presses the button, a SMS with her/his exact location is sent to the assistant.

The SMS received from the B/VIP is formatted in such a way that when the assistant receives the SMS, with a simple click (touch) on the received text, the Google Maps application opens, showing the exact location of the B/VIP, thus, creating the possibility to generate a route to it and therefore the B/VIP can be reached very fast.

The data received on the Arduino board from the Sim 808 module are of the String type, int the form of a single variable, data separated by the "," character, of the following form:

_CGNSINF: <GNSS run status>, <Fix status>, <UTC date \& Time $>,<$ Latitude $>,<$ Longitude $>, \quad<M S L$ Altitude>,<Speed Over Ground>, <Course Over Ground $>$, <Fix Mode $>,<R e s e r v e d 1>,<H D O P>$, $<P D O P>$, $<V D O P>$, $<$ Reserved2 $>$, $<$ GNSS Satellites in View $>$, $<$ GNSS Satellites Used $>,<G L O N A S S$ Satellites Used $>,<$ Reserved3 $>,<$ C/NO max $>,<H P A>,<V P A>O K$

In order to send the formatted SMS so that the assistant receives the exact location of the B/VIP on Google Maps, we extract from the String received in two separate strings the latitude and longitude information. In the program, a String vector called the date is declared. Each character in the String received from the way is red and then added to the vector and at the character encounter "," the variable $i$ is incremented, so each element in the vector contains separate data.

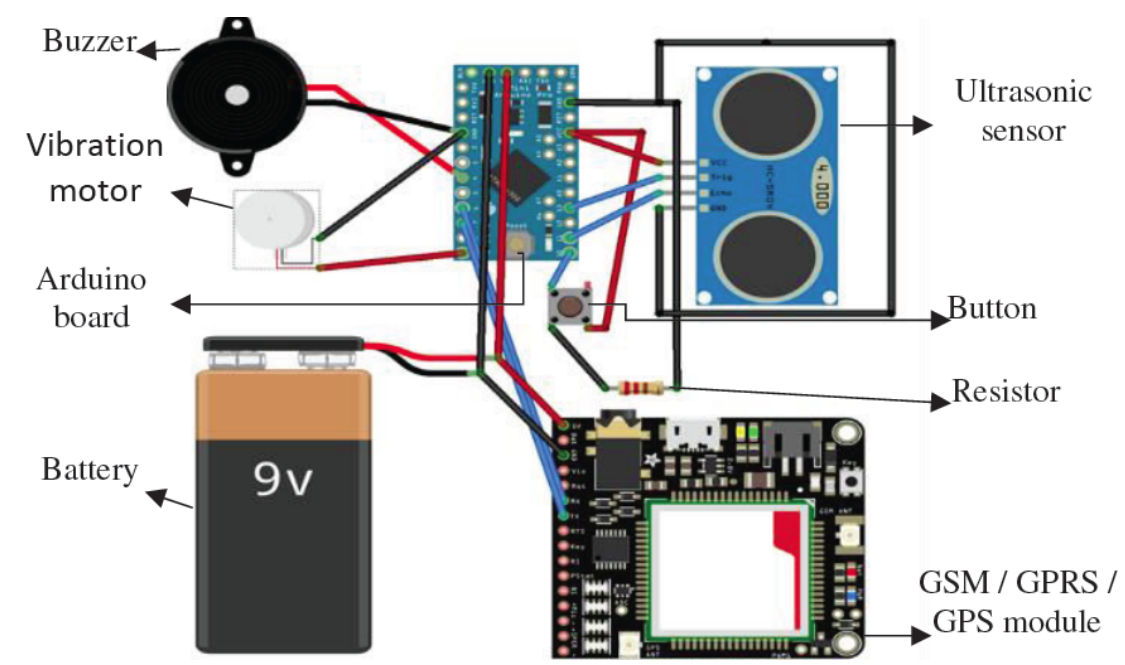

Figure 3: The diagram of the module with GPS. 
The SMS sent to the assistant is as follows:

"http://maps.google.com/maps?q=loc:"+latitude+","+lon gitude

The hardware implementation of the project consists in assembling and configuring all the components presented above in an appropriate way so that they properly work together.

Figure 4 shows how to make the basic prototype located in the B/VIP's palm, a model after which the other prototypes are made.

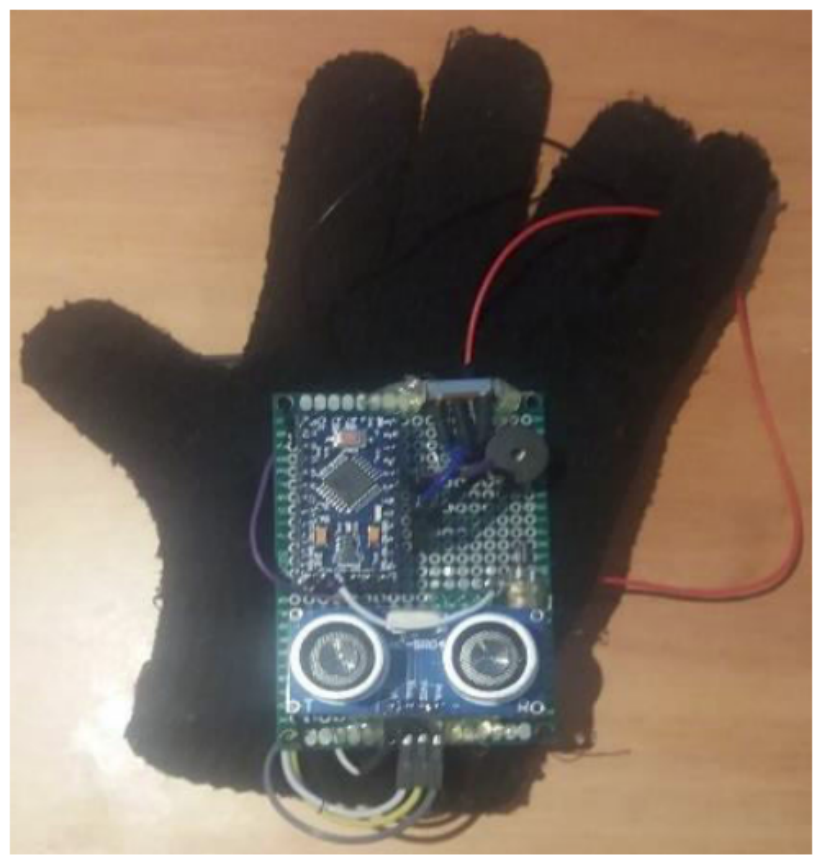

Figure 4: The module placed on to the glove in B/VIP's palm.

Figure 5 shows the hardware implementation of the prototype with the GSM / GPRS / GPS module. Because it is larger and heavier than the other modules, is aimed to be placed on the B/VIP left and/or right arm, secured with an elastic band.

Following tests conducted, since the B/VIP are able to move into different spaces (indoor or outdoor), the entire system has low weight, long time efficient, lowcost and handy, we have concluded that the system has potential to significantly improve B/VIP independency and also increase their self-confidence since they can more safe even in unknown spaces or environments.

\section{CONCLUSIONS}

The system addressed in this paper represents a first step in using off-the-shelf, low cost spare parts,

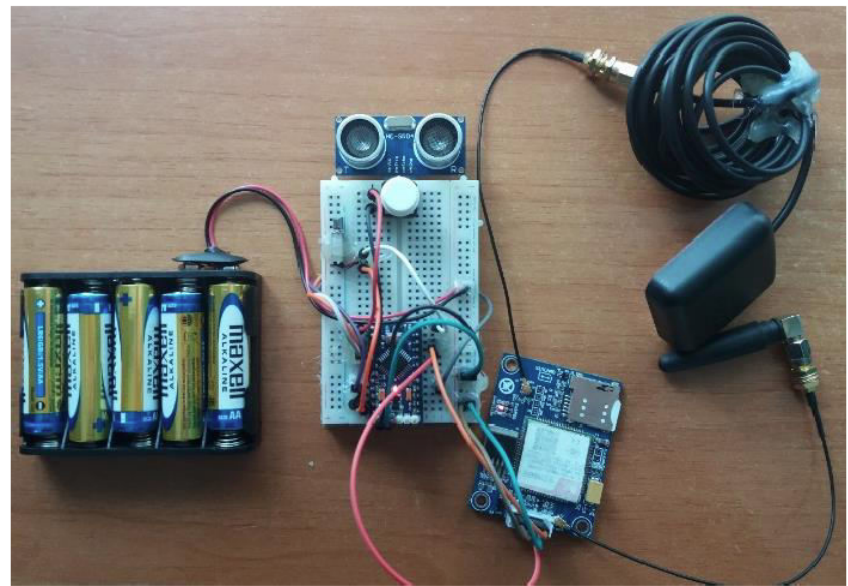

Figure 5: The module placed on B/VIP's (left on right) arm.

based on current technology. It aims to improve the living standard of people with visual disabilities, thus allowing them to increase their mobility both indoor and outdoor and therefore their confidence and independency. We believe that the use of this system by the B/VIP can improve their mobility level in a very good way, because the system can be configured according to their needs and improved, as well. Moreover, wearing this system gives the possibility to $\mathrm{B} / \mathrm{VIP}$ to use hands, therefore, a cane or a guide dog, can be additionally wear. If B/VIP choose to mount the system on different objects in the house, he/she knows in due time, from higher distance where the obstacle is and how close the object is. However, one of the most important functions of the system is the GPS tracking, so the B/VIP can safely go to different locations of interest. In this way, the situation when the B/VIP can no longer reach home and does not know where he/she is avoided. With a simple press of a button the caretaker finds him/her out in a fast way and the precise location. Thinking to further improvements, different other sounds or vibrations, can be added as new functionality, in order to guide B/VIP to different points of interest and back home. Another aspect to be pointed out regarding this system is its cost of production, being a very low one compared many others. For example, the demand for guide dogs cannot be fully assured because the number of such animals is limited by factors that cannot be influenced by humans. On the other hand, different other smart devices, similar with the one presented here, cannot fully satisfy the B/VIP's necessities or are too expensive. Based on the principles presented, the developed system is a first implementation with a "rough" design, but an intelligent, advance assistance system dedicated to B/VIP, that is possible to be further developed with low cost. 


\section{REFERENCES}

[1] Zöllner M, Huber S, Jetter HC, Reiterer H. NAVI - A Proof-ofConcept of a Mobile Navigational Aid for Visually Impaired Based on the Microsoft Kinect. In: Campos P, Graham N, Jorge J, Nunes N, Palanque P, Winckler M. (eds) HumanComputer Interaction - INTERACT 2011. INTERACT 2011. Lecture Notes in Computer Science, vol 6949. Springer, Berlin, Heidelberg, 2011. https://doi.org/10.1007/978-3-642-23768-3 88

[2] Bradley NA, Dunlop MD. An experimental investigation into wayfinding directions for visually impaired people. Personal Ubiquitous Computing 2005; 9: 395-403. https://doi.org/10.1007/s00779-005-0350-y

[3] Cassinelli A, Reynolds C, Ishikawa M. Augmenting spatial awareness with haptic radar. In: Cruz I, Decker S, Allemang D, Preist C, Schwabe D, Mika P, Uschold M, Aroyo LM, Eds. ISWC 2006. LNCS, Springer, Heidelberg 2006; vol. 4273: pp. 61-64.

[4] Ross DA, Blasch BB. Wearable interfaces for orientation and wayfinding. In: Proc. ASSETS 2000, ACM, New York 2000; pp. $193-200$ https://doi.org/10.1145/354324.354380

[5] https://www.who.int/news-room/fact-sheets/detail/blindnessand-visual-impairment, last accessed in $22^{\text {nd }}$ of November, 2019

[6] Riazi A, Riazi F, Yoosfi R, Bahmeei F. Outdoor difficulties experienced by a group of visually impaired Iranian people. In: J Curr Ophthalmol 2016; 28(2): 85-90. https://doi.org/10.1016/j.joco.2016.04.002

[7] Koestler FA. Historical Chronologies, The Unseen Minority: A Social History of Blindness in the United States, In: American Printing House for the Blind 2012.

[8] https://www.visionaware.org/info/everyday-living/essentialskills/an-introduction-to-orientation-and-mobility-skills/dogguides-for-people-with-vision-loss/1234, last accessed in $22^{\text {nd }}$ of November, 2019
[9] Bailly C, Les débuts de la canne blanche, l'Auxilaire des aveugles 1990.

[10] Nivedita A, Sindhuja M, Asha G, Subasree RS, Monisha S. Smart cane navigation for visually impaired, International Journal of Innovative Technology and Exploring Engineering (IJITEE) ISSN: 2278-3075, 2019; Volume-8 Issue-6S.

[11] Putnam PB. Love in the Lead: The Miracle of the Seeing Eye Dog (2nd ed.). University Press of America. 1997; p. 20.

[12] https://www.dailymail.co.uk/sciencetech/article2659993/Smart-glasses-BLIND-Device-transforms-worldoutlines-shapes-help-partially-sighted-navigate.html, last accessed in $22^{\text {nd }}$ of November, 2019

[13] https://noizz.ro/big-stories/tandem-acces-aplicatia-careghideaza-nevazatorii-prin-metrou/b6r00tg last accessed in $22^{\text {nd }}$ of November, 2019

[14] Hogle P. A New Take on Augmented Reality: Cydalion Navigation App Aids People with Low Vision, November 08, 2016, www.learningsolutionsmag.com/articles/2129

[15] Riurean S, Leba M, Ionica A. Indoor Positioning and Guiding System Based on VLC for Visually Impaired People, DSIC'19 - The International Conference on Digital Science, Limassol, Cyprus, 2019.

[16] Perez-Jimenez R, Rabadan J, Guerra V, Aguiar L, Rufo J. Fundamentals of Indoor VIp: Providing Autonomous Mobility for Visually Impaired People, International Conference and Workshop on Bioinspired Intelligence (IWOBI), Funchal, 2017; 2017: pp. 1-6. https://doi.org/10.1109//WOBI.2017.7985542

[17] Kasthuri R, et al. Smart Device for Visually Impaired People, Third International Conference on Science Technology Engineering \& Management (ICONSTEM) 2017. https://doi.org/10.1109/ICONSTEM.2017.8261257

[18] Purdum JJ. Beginning C for Arduino: learn C programming for the Arduino Second ed. Apress New York 2015. https://doi.org/10.1007/978-1-4842-0940-0

[19] https://www.migatron.com/distance-measurement-sensors/, last accessed in 22nd of November, 2019.

\section{DOI: https://doi.org/10.31875/2409-9694.2019.06.4}

(C) 2019 Costache et al.; Zeal Press

This is an open access article licensed under the terms of the Creative Commons Attribution Non-Commercial License (http://creativecommons.org/licenses/by-nc/3.0/) which permits unrestricted, non-commercial use, distribution and reproduction in any medium, provided the work is properly cited. 